\title{
Constraints of hadronic interaction models from the cosmic muon observations
}

\author{
L.G. Dedenko ${ }^{1,2, a}$, A.V. Lukyashin ${ }^{1, b}$, G.F. Fedorova ${ }^{2, c}$, and T.M. Roganova ${ }^{2, d}$ \\ 1 Faculty of Physics M.V. Lomonosov Moscow State University, Leninskie Gory, 119991 Moscow, Russia \\ 2 Skobeltsin Institute of Nuclear Physics, Lomonosov Moscow State University, 119234 Moscow, Russia
}

\begin{abstract}
A simple method of the vertical muon energy spectrum simulations has been suggested. These calculations have been carried out in terms of various models of hadronic interactions. The most energetic $\pi^{ \pm}$-mesons and $\mathrm{K}^{ \pm}$-mesons produced in hadron interactions contribute mainly to this energy spectrum of muons due to the very steep energy spectrum of the primary particles. So, some constraints on the hadronic interaction models may be set from a comparison of calculated results with cosmic data on the vertical muon energy spectrum. This comparison showed that the most energetic secondary particles production is too high in the case of the QGSJET II-04 model and rather low in the case of the QGSJET II-03 model. These conclusion have been supported by the LHC data.
\end{abstract}

\section{Introduction}

The longitudinal development of extensive air showers (EAS), in particular, the depth $X_{\max }$ of its maximum, is determined by the rate of fragmentation of energy $E_{0}$ of the primary particle. This rate depends on the interaction cross sections of shower particles, and on the energy spectra of secondary particles are generated in interactions. Obviously, if the probability of particle production with energies close to the energy of the incident particle is high then the development of the cascade slows down. Conversely, in the case of rapid fragmentation of the incident energy cascades develop rather rapidly. The depth $X_{\max }$ of shower maximum in many studies is the main parameter for determining the composition of primary cosmic radiation (PCR) at ultra-high energies. It should also be noted that in the case of the slow rate of development cascade and hence large values of depth $X_{\max }$ the lateral distribution of the shower particles at the observation level becomes narrower. Therefore, the values of signals in the surface and underground detectors located at large distances from the shower core are decreased. It must be taken into account when determining the density of muons at large distances from the shower core and the composition of the PCR found from the muon lateral distribution. Studies of the composition and characteristics of the energy spectrum of the PCR are important components of theories of the origin of cosmic rays at ultra-high energies. Interpretation of experimental data on the depth of the shower maximum $X_{\max }$ and the observed fraction of muons at a fixed distance from the shower core are carried out in terms of different hadronic

\footnotetext{
a e-mail: ddn@dec1.sinp.msu.ru

b e-mail: lukyashin.anton@physics.msu.ru

c e-mail: fdr@dec1.sinp.msu.ru

d e-mail: rogatm@yandex.ru
}

interaction models. In the case of "soft" interaction the generation of secondary particles emitted at mostly small angles with respect to the projectile particle (the highest values of pseudorapidity $\eta$ ) is of importance. The models based on the Gribov-Regge theory [1,2] are commonly used. The dominant contribution of the pomeron at ultrahigh energies and other effects are taken into account differently in various models [3-5]. Therefore, testing models at the highest energies of secondary particles is very important for understanding the physics of hadron interactions and for the interpretation of the EAS data. At the LHC this testing is carried out in the LHCf [6] and TOTEM [7] experiments. In cosmic rays, we suggest testing of models of hadron interactions with the help of the atmospheric muon spectrum. In this case, due to the higher slope of the energy spectrum of the primary particles (a coefficient of the slope of the difference spectrum is $\gamma=2.75$ ) the generation of secondary particles $\left(\pi^{ \pm}\right.$-mesons and $K^{ \pm}$-mesons) with the highest energies is of importance. It was shown [8] that the QGSJET II-03 model [4] leads to a spectrum of vertical muons, the intensity of which is about a factor $f=1.5$ times less than the data of collaborations L3 + Cosmic [9], MACRO [10] and LVD [11]. The result [8] was obtained as a solution of transport equations. The $\pi^{ \pm}$ and $K^{ \pm}$mesons decay into $\mu^{ \pm}$-mesons. These $\pi^{ \pm}$and $K^{ \pm}$ mesons are generated by the parent particles of several generations. Therefore, if some excess of the secondary particle production in the singular interactions of hadrons is determined by the coefficient $k$, then, in the case of $i$ generations, the factor $f$ will be $f \approx k^{i}$. Thus, the spectra of atmospheric muons are a very useful tool to test the models of hadronic interactions. However, other factors (cross sections of interactions, etc.) affect the result of comparison. In this article we propose to test the hadron interaction models by a very simple original method [12] with the help of observed atmospheric muon spectrum. 


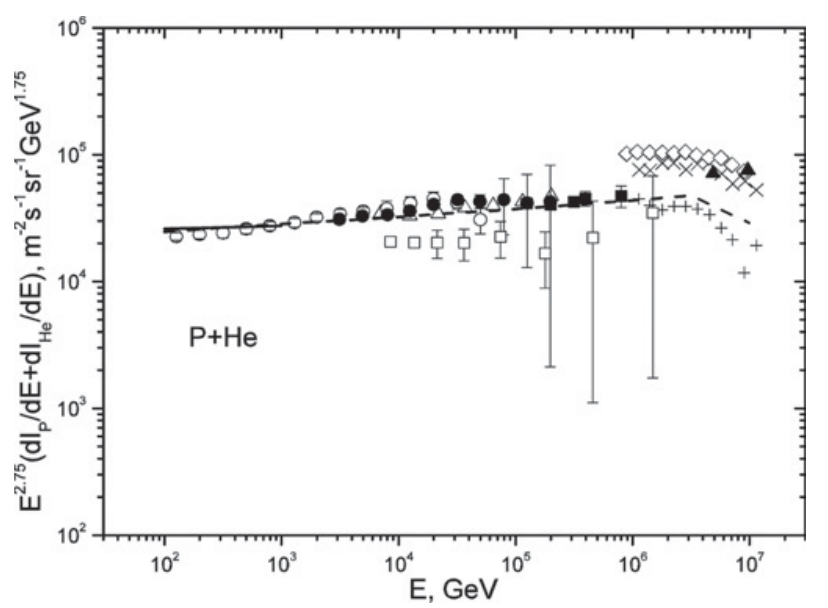

Figure 1. The primary spectrum of protons and helium nuclei $(\mathrm{p}+\mathrm{He})$. Dashed line - modified Gaisser-Honda [13] approximation. Data: solid line - AMS02 [14]; ○ ATIC 2 [15];

- CREAM [16]; 口 WCFTA [17]; $\triangle$ ARGO [18]; $\square$ RUN JOB [19]; $\diamond$ TUNKA [21] (all particles); $\triangle$ SPHERE2 2 [22] (all particles); $\times$ KASKADE [20] (all particles, QGSJET II-03); + KASKADE [20] (all particles, SIBYLL 2.1).

\section{Method of calculation}

The very simple original method of simulations of the energy spectrum of vertical muons can be described as follow [12]. Let the $\left(d I_{p} / d E\right)$ and $\left(d I_{H e} / d E\right)$ be the differential energy spectra of the primary protons and helium nuclei. As for the spectrum of muons the energy per nucleon is of importance. So, the heavier nuclei give a negligible contribution to this spectrum. In the energy range of $10^{2}-3 \cdot 10^{6} \mathrm{GeV}$, we used the approximations $\left(d I_{p} / d E\right)_{G H}$ and $\left(d I_{H e} / d E\right)_{G H}$ by Gaisser-Honda [13]. At energies above $E_{1}=3 \cdot 10^{6} \mathrm{GeV}$, these spectra were multiplied by a factor $\left(E_{1} / E\right)^{0.5}$ and are refered as modified GH approximation. Figure 1 shows a comparison of the sum of modified $\mathrm{GH}$ approximations [13] for primary protons and helium nuclei (dotted line) with the experimental data AMS02 [14] - solid line, ATIC2 [15] - hollow circles (o), CREAM [16] - dark circles (•), WCFTA [17] - dark squares (ם), ARGO [18] - hollow triangles $(\triangle)$, RUN JOB [19] - hollow squares $(\square)$. The experimental data KASKADE [20], interpreted in terms of the model of QGSJET II-03 shows the oblique crosses $(x)$, and in terms of the model SIBYLL 2.1 - straight crosses (+). The experimental data TUNKA [21] for all primary particles are shown by hollow diamonds $(\diamond)$, and data SPHERE 2 [22] - dark triangles $(\boldsymbol{\Delta})$. The spectra of the primary protons and primary helium nuclei are presented separately in Fig. 2 and Fig. 3 with various data mentioned above for a comparison. The notation: $(\boldsymbol{\Delta})$ is applied in Fig. 2 and Fig. 3 only for the data of AMS02 [14]. From comparison with data it can be concluded that the accepted approximation [13] does not overestimate the flux of the primary protons and helium nuclei. This is important for the conclusions on possible uncertainties of tested models. The energy spectra of vertical muons $D_{p}\left(E_{\mu}\right) d E_{\mu}$ and $D_{H e}\left(E_{\mu}\right) d E_{\mu}$ induced by the primary protons and helium nuclei are expressed by simple integrals over the energy $E$

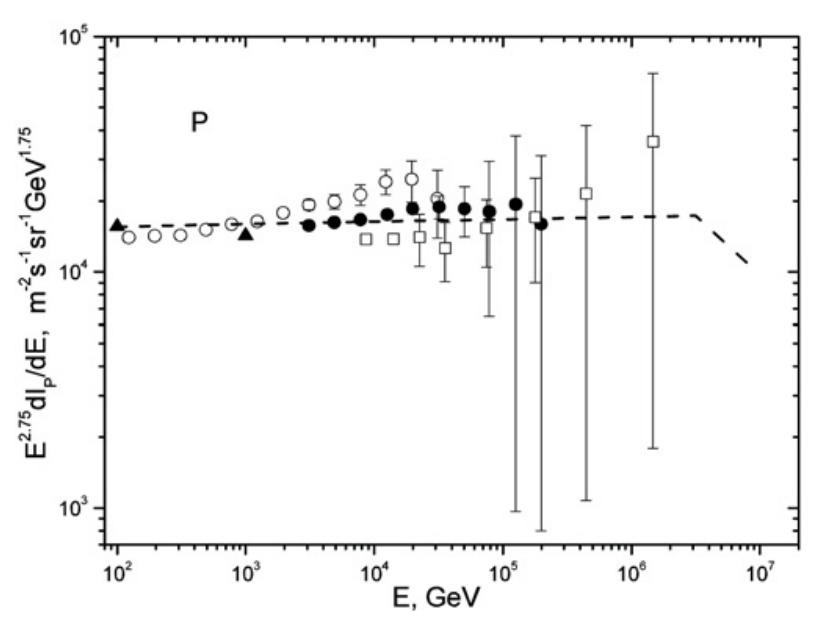

Figure 2. The energy spectrum of the primary protons. Dashed line - modified Gaisser-Honda [13] approximation. Various data - see the text. $\triangle$ AMS02 [14]; ○ ATIC 2 [15]; • CREAM [16]; $\square$ RUN JOB [19].

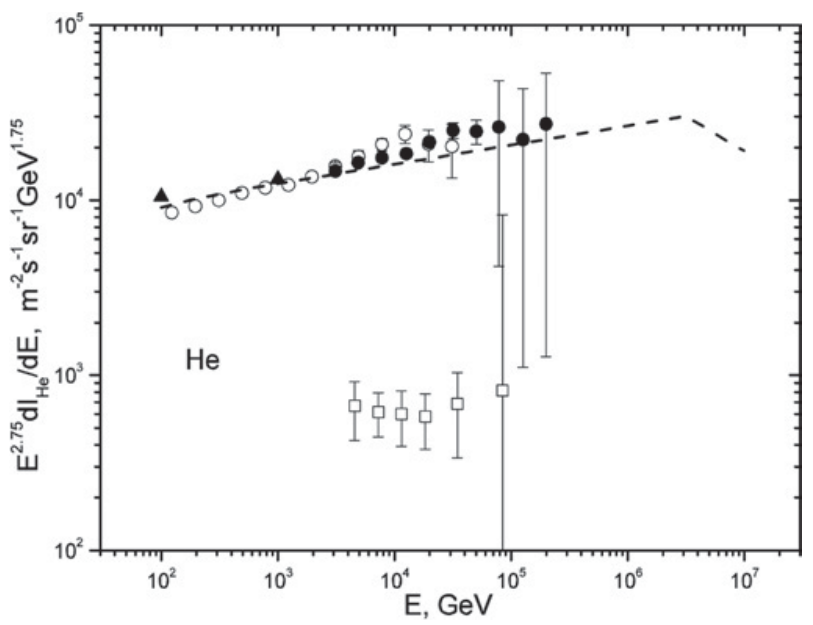

Figure 3. The energy spectrum of the primary helium nuclei. Dashed line - modified Gaisser-Honda [13] approximation. Various data - see the text. A AMS02 [14]; ○ ATIC 2 [15]; - CREAM [16]; $\square$ RUN JOB [19].

of the primary particles as follows:

$$
\begin{gathered}
D_{p}\left(E_{\mu}\right) \cdot d E_{\mu}=\int d E \cdot\left(\frac{d I_{p}}{d E}\right) \cdot S_{p}\left(E_{\mu}, E\right) \cdot d E_{\mu} \\
D_{H e}\left(E_{\mu}\right) \cdot d E_{\mu}=\int d E \cdot\left(\frac{d I_{H e}}{d E}\right) \cdot S_{H e}\left(E_{\mu}, E\right) \cdot d E_{\mu} .
\end{gathered}
$$

The sum of these spectra:

$$
D\left(E_{\mu}\right)=\left(D_{p}\left(E_{\mu}\right)+D_{H e}\left(E_{\mu}\right)\right)
$$

will be used for comparison with data [9-11]. Functions $S_{p}\left(E_{\mu}, E\right) \cdot d E_{\mu}$ and $S_{H e}\left(E_{\mu}, E\right) \cdot d E_{\mu}$ are the differential energy spectra of muons in showers induced by primary protons and helium nuclei with fixed energy $E$. These spectra were calculated for 24 and 19 values of the energy $E$ of the primary protons and helium nuclei, respectively, in the range of $10^{2} \div 10^{7} \mathrm{GeV}$. Calculations 


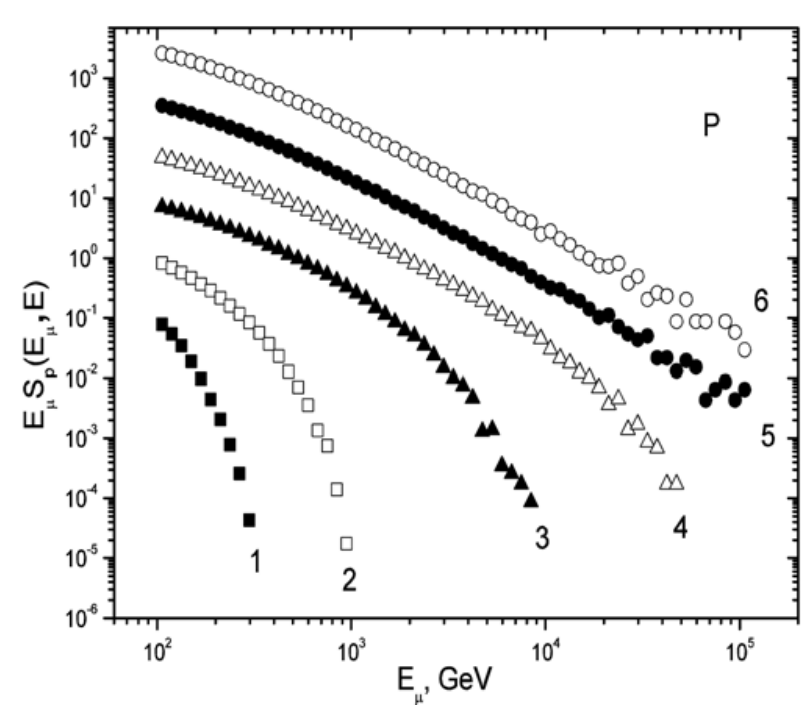

Figure 4. The energy spectra of muons in showers induced by primary protons with various fixed energies $E$ (simulations in terms of QGSJET II-04 model): $1-3,162 \cdot 10^{2} ; 2-10^{3} ; 3-10^{4} ; 4$ $10^{5} ; 5-10^{6} ; 6-10^{7} \mathrm{GeV}$.

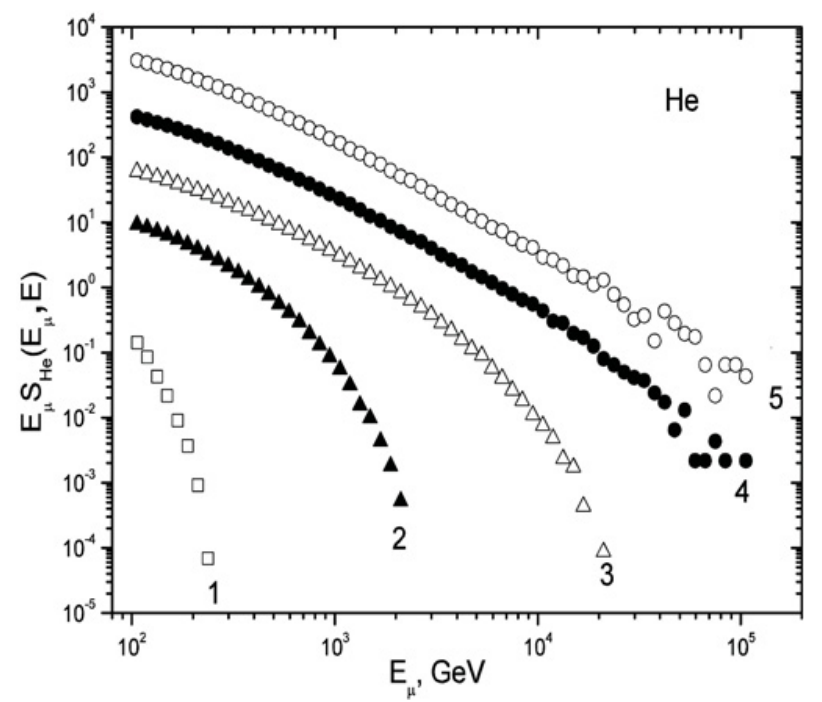

Figure 5. The energy spectra of muons in showers induced by primary helium nuclei with various fixed energies $E$ (simulations in terms of QGSJET II-04 model): $1-10^{3} ; 2-$ $10^{4} ; 3-10^{5} ; 4-10^{6} ; 5-10^{7} \mathrm{GeV}$.

have been carried out in terms of two models of hadron interactions (QGSJET II-03 [4] and QGSJET II-04 [5]) using the package CORSIKA 7.4 [23]. The calculations were performed with statistics of $10^{6}$ events in the low energy region and up to $10^{2}$ events at the highest energies of the primary particles.

Figure 4 and Fig. 5 show examples of the muon energy spectra $S_{p}\left(E_{\mu}, E\right)$ and $S_{H e}\left(E_{\mu}, E\right)$ in the energy range of $10^{2} \div 10^{5} \mathrm{GeV}$ calculated in terms of the QGSJET II04 model [5] for 6 values of proton energies and 5 values of helium nuclei energies, respectively. It is seen that in the energy range of $10^{4} \div 10^{6} \mathrm{GeV}$ statistics is small. Therefore, we will use the energy range of $10^{2} \div 10^{4} \mathrm{GeV}$ for a comparison. Dependence of the spectra $S_{p}\left(E_{\mu}, E\right)$

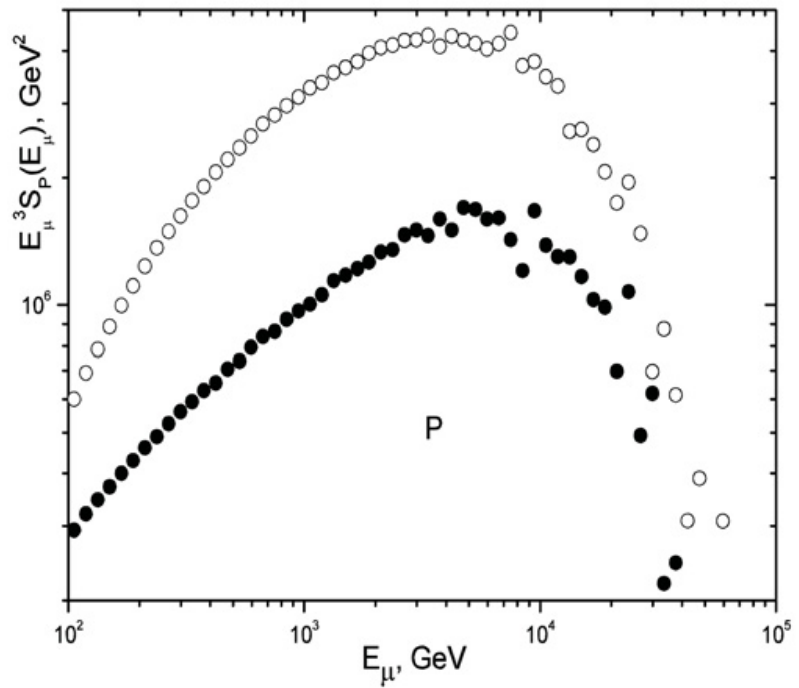

Figure 6. The energy spectra of muons in showers induced by primary protons with fixed energy $E=10^{5} \mathrm{GeV}$ calculated in terms of two models: • QGSJET II-03, ○ QGSJET II-04.

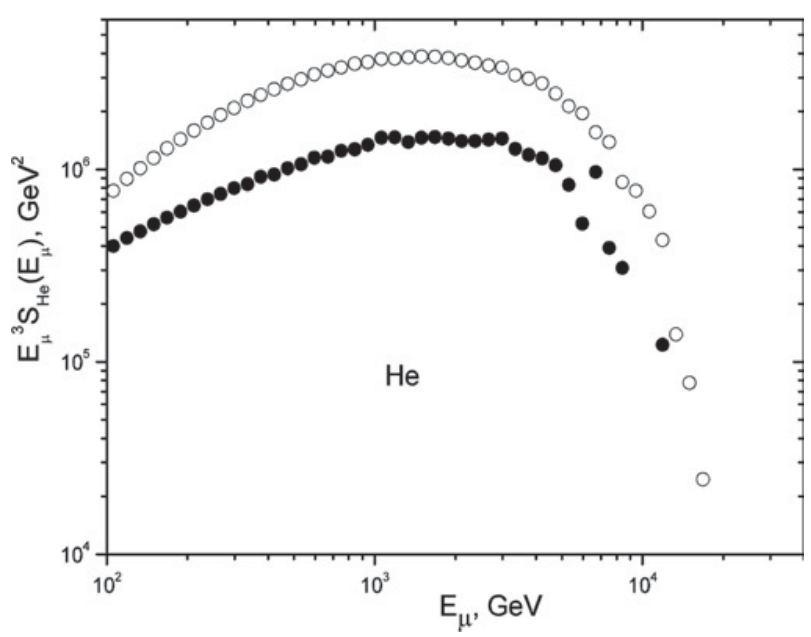

Figure 7. The energy spectra of muons in showers induced by primary helium nuclei with fixed energy $E=10^{5} \mathrm{GeV}$ calculated in terms of two models: • QGSJET II-03, ○ QGSJET II-04.

and $S_{H e}\left(E_{\mu}, E\right)$ on the models of hadron interactions (QGSJET II-03 (•) and QGSJET II-04 (०)) are shown in Fig. 6 and Fig. 7 for the primary protons and helium nuclei, respectively for the energy $E=10^{5} \mathrm{GeV}$. It is evident that the model QGSJET II-04 [5] predicts the greatest density of muons, while the model QGSJET II-03 [4] - the lowest one.

\section{The results of simulations}

The spectra of vertical muons $D\left(E_{\mu}\right)$ in the energy range of $10^{2}-10^{4} \mathrm{GeV}$ for the hadron interaction models QGSJET II-03 [4] and QGSJET II-04 [5] are presented in Fig. 8. It can be seen that the QGSJET II-04 model [5] predicts the intensity of muon flux by a factor $2 \div 3$ higher than the intensity of the muon flux calculated in terms of the QGSJET II-03 [4] model. This finding is consistent with the results shown in Fig. 6 and Fig. 7. Figure 8 also very clearly demonstrates an increase in the steepness 


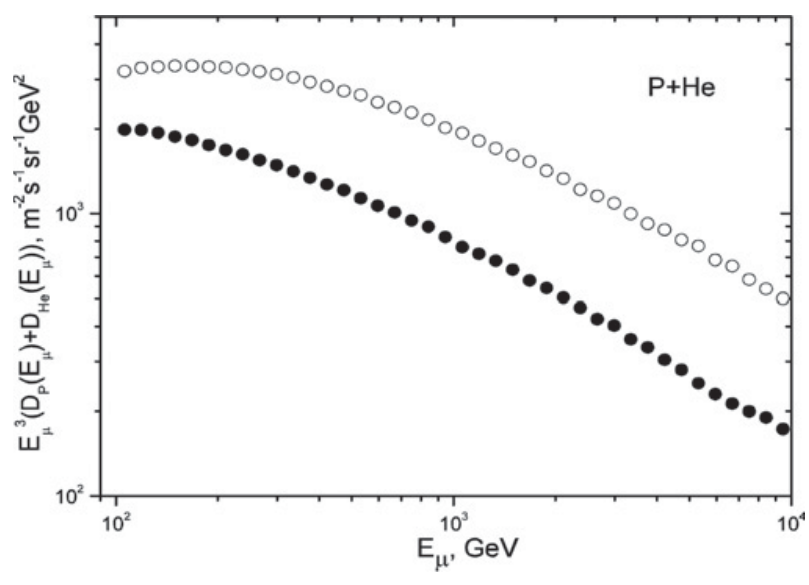

Figure 8. The calculated energy spectra of near vertical muons for various models: • QGSJET II-03, 。 QGSJET II-04.

of the muon spectrum at energies above $100 \mathrm{GeV}$. The constant $B_{\pi} \simeq 100 \mathrm{GeV}$ is the decay constant for $\pi$-mesons in the atmosphere. Therefore, the muon spectrum becomes steeper because $\pi$-mesons at energies $E>B_{\pi}$ rather interact with nuclei in the atmosphere than decay into muons. Comparison of the calculated spectra with experimental data allows us to test models. The ratios MC/DATA of the results of calculations for models [4] and [5] to the smooth approximation of the data of collaborations L3 + Cosmic [9], MACRO [10] and LVD [11] are shown in Fig. 9. It can be seen that these ratios are increasing from $\sim 1.4$ to $\sim 1.7$ for the QGSJET II04 model [5] and are decreasing from $\sim 0.8$ to $\sim 0.6$ for QGSJET II-03 model [4] at muon energies in the range of $10^{2} \div 10^{4} \mathrm{GeV}$. The most important fact is that these increase becomes higher at energies, $E_{\mu}$, above $10^{3} \mathrm{GeV}$ for the QGSJET II-04 model. For the QGSJET II-03 model the ratios MC/DATA become constant at the level of $\sim 0.6$ for energies $E_{\mu}$ above $10^{3} \mathrm{GeV}$. No slowing of this increase is seen at higher muon energies. Thus, Fig. 9 demonstrates a very serious difference between the calculated spectra and the data reported in [9-11]. This difference is associated with a different rate of energy fragmentation of projectile particles in events of its interactions with nuclei in the atmosphere. Thus, the QGSJET II-03 model underestimate the probability of secondary particle production at the highest energies. The QGSJET II-04 model overestimate this probability of secondary particle production at the highest energies. According to calculations, the main contribution to the integrals $D_{p}\left(E_{\mu}\right) \cdot d E_{\mu}$ and $D_{H e}\left(E_{\mu}\right) \cdot d E_{\mu}$ comes from secondary particles with energies in the ranges of $(0.01-0.6) E$ and $(0.001-0.1) E$, where $E$ is the energy of a projectile particle for primary protons and helium nuclei, respectively. The results of this comparison are also confirmed by the data of LHCf [6] and TOTEM [7].

For example, the QGSJET II-04 model [5] overestimates the density of charged particles $d N_{c h} / d \eta$ per unit of pseudorapidity at the pseudorapidity $\eta=6.345$ by a factor of $k \approx 1.3$ as compared to the TOTEM data [7]. This difference increases at large values $\eta$ because of the difference between the slopes of the calculated curve and the data from [7]. The QGSJET II-04 [5] model predicts

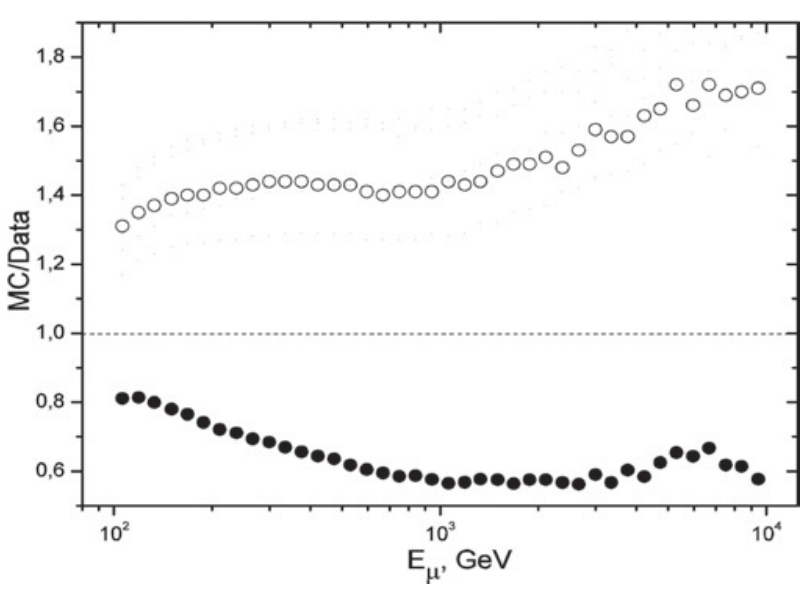

Figure 9. Comparison of calculated muon energy spectrum with data [9-11]. The ratios MC/DATA are shown: • QGSJET II-03, 。 QGSJET II-04.

the density $d N_{c h} / d \eta$ which is (18-30)\% higher than the data [7] in the range $5.3 \leq \eta \leq 6.4$. It is also important to note that for differences of rapidity $\Delta y \simeq 0$, the value of the average transverse momentum $\left\langle p_{T}\right\rangle$ for $\pi^{0}$-mesons is about $50 \mathrm{MeV} / \mathrm{c}$ less than data [24]. It is assumed that a similar decreasing of the $\left\langle p_{T}\right\rangle$ dependence is also valid for charged $\pi$ mesons. The calculated density of muons at large distances from the shower axis will be underestimated. This underestimation was observed by the Pierre Auger Collaboration [25] and in Yakutsk [26]. The energy spectra of photons in $p-p$ collisions at the energy of $\sqrt{s}=7 \mathrm{TeV}$ in the pseudorapidity range $8.81 \leq$ $\eta \leq 8.99$ are $2 \div 4$ times above those predicted by the QGSJET II-03 model [4]. So, all these models should be significantly corrected at the highest energies of secondary particles.

\section{Conclusion}

The atmospheric muon energy spectra calculated in terms of the QGSJET II-04 [5] model is by a factor $f=1,7$ higher then data [9-11] at energy $E_{\mu}=10^{4} \mathrm{GeV}$. The atmospheric muon energy spectrum calculated in terms of the QGSJET II-03 model [4] is by a factor $f=1,5$ lower then data [9-11] at energy $E_{\mu}=10^{4} \mathrm{GeV}$. So, we can conclude, these models of hadronic interactions should be updated for very high energies of secondary particles.

The authors thank LSS grant (grant 3110.2014.2) for support! The speaker thanks the organizing committee of ISVHECRI 2014 for a well organised conference and administrative support!

\section{References}

[1] V. Gribov. Sov. Phys. JETP 26. 414 (1968)

[2] T.Regge. Nuovo Cimento 14, 951 (1959)

[3] E.-J. Ahn, R. Engel, T.K. Gaisser, P. Lipari, and T. Stanev, Phys. Rev. D 80, 094003 (2009)

[4] S.S. Ostapchenko, Phys. Rev. D 74, 014026 (2006) 
[5] S.S. Ostapchenko, Phys. Rev. D 83, 014018 (2011)

[6] H. Menjo, O. Adriani, and M. Bongi (for LHCf Collaboration), Nucl. Instrum. Methods Phys. Res. A 692, 224 (2012)

[7] G. Latino (on behalf of TOTEM Collab.), arXiv: hep-ex/1302.2098v1 (2013)

[8] A.A. Kochanov, T.S. Sinegovskaya, S.I. Sinegovsky, Astrop. Physics 30, 219 (2008)

[9] The L3 Collab., arXiv: hep-ex/0408114v1K (2004)

[10] M. Ambrosio, R. Antolini, G. Auriemma et al. (The MACRO Collaboration), Phys. Rev. D 52, 3793 (1995)

[11] M. Aglietta, B. Alpat, E.D. Alieva et al. (The LVD Collaboration), arXiv: hep-ex/9806001v1 (1998)

[12] L.G. Dedenko, S.P. Knurenko, A.K. Makarov, I.T. Makarov, M.I. Pravdin, I.Ye. Sleptsov, A.V. Glushkov, G.F. Fedorova, T.M. Roganova, and A.A. Sabourov, in Proceedings of the 33th International Cosmic Ray Conference (Rio-deJaneiro, 2013); https://143.107.180.38/ indico/contributionDisplay $\cdot$ py?contribId= 0045\&ses ion $I d=3 \& \operatorname{conf} I d=0$

[13] T. Gaisser and M. Honda, Ann. Rev. Nucl. Part. Sci. 52, 153 (2002)

[14] V. Choutko (on behalf AMS Collaboration), in Proceedings of the 33-th International Cosmic Ray Conference (Rio-de-Janeiro, 2013); https://143. 107.180.38/indico/contributionDisplay.py ? contribId=1262\&ses ion $I d=3 \& \operatorname{conf} I d=0$

[15] A.D. Panov, J.H. Adams Jr., and H.S. Ahn (for ATIC-2 Collab.), Bull. Russ. Acad. Sci.: Phys. 71, 494 (2007); A.D. Panov, J.H. Adams Jr., H.S. Ahn, et al., Bull. Russ. Acad. Sci.: Phys. 73, 564 (2009)

[16] H.S. Ahn (for the CREAM Collaboration), Astrophys. J. Lett. 714, L89 (2010)
[17] S.S. Zhang (for the WFCTA Collaboration), Nucl. Instrum. Methods Phys. Res. A 629, 57 (2011)

[18] B. Bartoli (for the ARGO_YBJ Collaboration), Phys. Rev. D 85, 092005 (2012)

[19] V.A. Derbina, V.I. Galkin, and M. Hareyama (for the RUNJOB Collaboration), Astrophys. J. 628, L 41 (2005)

[20] T. Antoni (for the KASCADE Collaboration), Astropart. Phys. 24, 1 (2005)

[21] V.V. Prosin (on behalf TUNKA Collaoration.), in Proceedings of the 33th International Cosmic Ray Conference (Rio-de-Janeiro, 2013); https://143. 107.180.38/indico/contributionDisplay.py ? contribId=0617\&session $I d=3 \& \operatorname{conf} I d=0$

[22] R.A. Antonov, T.V. Aulova, S.P. Beschapov, E.A. Bonvech, D.V. Chernov, T.A. Dzhatdoev, Mir. Finger, Mix. Finger, V.I. Galkin, N.V. Kabanova, A.S. Petkun, D.A. Podgrudkov, T.M. Roganova, S.B. Shaulov, and T.I. Sysoeva, in Proceedings of the 33th International Cosmic Ray Conference (Rio-de-Janeiro, 2013); https://143. 107.180.38/indico/contributionDisplay.py ? contribId=1185\&ses ion $I d=3 \& \operatorname{conf} I d=0$

[23] D. Heck, J. Knapp, J.-N. Capdevielle G. Schatz, and T. Thouw, Forschungszentrum Karlsruhe Technical Report No. 6019, Karlsruhe (1998)

[24] O. Adrian, L. Bonech, M. Bongi (for the LHCf Collaboration), Phys. Rev. D 86, 092001 (2012)

[25] R. Engel (for The Pierre Auger Collab.), arXiv: 0706. 1921 [astro-ph] 251 Testing models of hadron interactions (2007)

[26] A.V. Glushkov, I.T. Makarov, M.I. Pravdin, I.E. Sleptsov, D.S. Gorbunov, G.I. Rubtsov, and S.I. Troitsky, JETP Lett. 87, 190 (2008). Pis'ma v ZhETF 87 (4), 220 (2008) 